\title{
Neuroprotective mechanisms of miR-124 activating PI3K/Akt signaling pathway in ischemic stroke
}

\author{
CHANGMING WANG ${ }^{*}$, ZHIJIE WEI* , GUOHONG JIANG and HAIJUN LIU \\ Department of Neurology, The Affiliated Hospital of Zunyi Medical College, Zunyi, Guizhou 563003, P.R. China
}

Received June 27, 2016; Accepted March 14, 2017

DOI: $10.3892 /$ etm.2017.4424

\begin{abstract}
The neuroprotective mechanisms of miR-124 activating phosphoinositide 3-kinase (PI3K)/Akt signaling pathway in ischemic stroke were investigated. The oxygen-glucose deprivation model of nerve cells induced by PC12 cells was established in vitro, then miR-124 mimics or inhibitor was transfected and synthesized by liposome. Cells were divided into the blank control, model, mimics and inhibitor groups, and the apoptotic rate was determined using flow cytometry. Additionally, the expression levels of PI3K, Akt, Bax, Bcl-2, caspase-3 mRNA and protein were tested by quantitative PCR and western blot analysis at $0,3,6$, 12 and $24 \mathrm{~h}$, respectively. The apoptotic rate at each time-point in the blank control group was not significantly different. The apoptotic rate of the model and inhibitor groups increased over time, whereas the mimics group decreased $(\mathrm{P}<0.05)$. The apoptotic rate at each time-point in the mimics group was significantly lower than that of the model and inhibitor groups, and the rate of the inhibitor group was higher than that of the model group $(\mathrm{P}<0.05)$. PI3K, Akt and Bcl-2 mRNA and protein expression levels at the different time-points in the mimics group were significantly higher than those of the remaining groups $(\mathrm{P}<0.05)$. The expression levels of Bax and caspase-3 mRNA and protein in the inhibitor group were the highest, followed by the model and mimics groups, while that of the blank control group was the lowest $(\mathrm{P}<0.05)$. The results suggest that miR-124 participates in the neural protection of ischemic stroke by activating the PI3K/Akt signaling pathway.
\end{abstract}

\section{Introduction}

Apoptosis of ischemic stroke is a principal mechanism that participates in nerve injury and recovery (1). As a cascade

Correspondence to: Dr Guohong Jiang, Department of Neurology, The Affiliated Hospital of Zunyi Medical College, 149 Dalian Road, Zunyi, Guizhou 563003, P.R. China

E-mail: gzzyyxywcm@sina.com

*Contributed equally

Key words: miR-124, phosphoinositide 3-kinase/Akt signaling pathway, ischemic stroke, oxygen-glucose deprivation model, apoptosis rate, Bax, Bcl-2, caspase-3 reaction, apoptosis is regulated by intracellular genes and extracellular factors (2). miR-124 is abundantly expressed in neuronal cells (3). As the richest miRNAs, miR-124 accounts for $25-48 \%$ of total miRNAs in the brain (3). Additionally, it participates in a series of significant physiological activities including neuronal cell cycle regulation, cell differentiation, spinal cord development and the regulation of adult neurogenesis (4).

Previous findings showed that the anoxic environment of cerebral stroke can stimulate the stable upregulation of miR-124 expression, which is reported to be closely related to the phosphoinositide 3-kinase (PI3K)/Akt pathway (5). The PI3K/Akt signaling pathway is an important surviving and anti-apoptotic signal transduction pathway, which plays a significant biological function in cell apoptosis, survival, proliferation, cytoskeleton change and other activities (6). Ischemic brain injury can activate the neural stem cells (NSCs) that gather within the brain when in a resting state, and induce proliferation and differentiate to neurons, which plays a role in repairing the nerve function (7).

The aim of the present study was to examine the neuroprotective mechanisms of miR-124 activating the PI3K/Akt signaling pathway in ischemic stroke. The results showed that miR-124 activation of the PI3K/Akt signaling pathway was involved in the induction and differentiation of NSCs and serves as a potentially significant target for the treatment of ischemic stroke.

\section{Materials and methods}

Establishment of oxygen-glucose deprivation (OGD) model of nerve cells. The neuron-like PC12 cell (conserved by Cell Laboratory of The Affiliated Hospital of Zunyi Medical College) with the appropriate growth condition (cell volume $85-90 \%$ ) was selected and washed 3 times using Dulbecco's modified Eagle's medium (DMEM) without glucose containing $10 \%$ fetal bovine serum and $1.0 \mathrm{mmol} / \mathrm{l}$ sodium hydrosulfide for culture. The cell culture flask was placed in the anoxic tank and incubated at $37^{\circ} \mathrm{C}$ for $0,3,6,12$ and $24 \mathrm{~h}$.

miR-124 mimics or inhibitor was transfected and synthesized by liposome. The miR-124 expression vector was constructed by BLOCK-iT ${ }^{\mathrm{TM}}$ Pol II miR-RNAi expression vector kit with EmGFP, and then the miR-124 expression vector or negative control plasmid was transfected using Lipofectamine 2000 
Table I. Comparison of apoptotic rate (\%).

\begin{tabular}{|c|c|c|c|c|c|c|c|}
\hline Groups & 0 & $3 \mathrm{~h}$ & $6 \mathrm{~h}$ & $12 \mathrm{~h}$ & $24 \mathrm{~h}$ & F-value & P-value \\
\hline Blank & $0.06 \pm 0.01$ & $0.08 \pm 0.01$ & $0.09 \pm 0.02$ & $0.10 \pm 0.02$ & $0.08 \pm 0.02$ & 0.635 & 0.427 \\
\hline Model & $32.6 \pm 10.3$ & $43.6 \pm 15.2^{\mathrm{a}}$ & $49.7 \pm 18.4^{\mathrm{a}}$ & $56.3 \pm 19.3^{a}$ & $64.8 \pm 20.3^{a}$ & 9.468 & $<0.001$ \\
\hline Mimics & $15.3 \pm 5.2$ & $12.6 \pm 5.5^{\mathrm{b}}$ & $10.5 \pm 5.6^{\mathrm{b}}$ & $9.4 \pm 4.3^{\mathrm{b}}$ & $8.6 \pm 4.0^{\mathrm{b}}$ & 8.352 & $<0.001$ \\
\hline Inhibitor & $40.5 \pm 12.6$ & $45.9 \pm 16.2^{\mathrm{a}}$ & $54.2 \pm 15.9^{\mathrm{a}}$ & $59.7 \pm 21.2^{\mathrm{a}}$ & $66.5 \pm 24.6^{a}$ & 9.123 & $<0.001$ \\
\hline F-value & 12.306 & 14.524 & 16.529 & 20.321 & 25.426 & & \\
\hline P-value & $<0.001$ & $<0.001$ & $<0.001$ & $<0.001$ & $<0.001$ & & \\
\hline
\end{tabular}

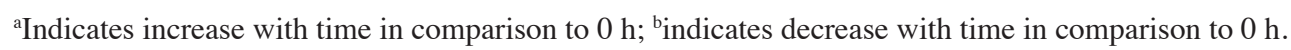

reagent (both from Invitrogen Life Technologies, Carlsbad, CA, USA) into the PC12 cell. The negative control plasmid contained a sequence that can form a similar precursor miRNA. Twenty-four hours after transfection, $3 \mathrm{nnsf}$ blasticidin (Sigma-Aldrich, St. Louis, MO, USA) was added to the culture media for continuous screening for 10 days. The resistant clones were selected using a fluorescence microscope and thereafter, the clones were expanded and re-cultured.

Experimental grouping. Cells were divided into four groups: Blank control group (PC12 cell), model group (PC12 cell OGD model), mimics group and inhibitor group, and the apoptotic rate was determined using flow cytometry (FCM). The expression levels of p-PI3K, p-Akt, Bax, Bcl-2, caspase-3 mRNA and protein were examined using RT-PCR and western blotting, at $0,3,6,12$ and $24 \mathrm{~h}$.

FCM technique. The cells were suspended in PBS and centrifuged at $850 \mathrm{x} g$ for $5 \mathrm{~min}$. Annexin V-FITC combination liquid $(500 \mu \mathrm{l})$ was added into each tube to resuspend the cells, followed by $5 \mu \mathrm{l}$ Annexin V-FITC and $10 \mu \mathrm{l}$ PI dye liquor (all from Beijing Zhongshan Biological Technology Co., Ltd., Beijing, China). After gentle mixing, the cells were incubated for $10 \mathrm{~min}$ at room temperature in the dark, and the apoptotic rate was determined by FCM (Beckman Coulter, Inc., Brea, CA, USA).

qPCR test. Total RNA was extracted by miRNA isolation kit, and the RNA was removed by DNase I (both from Ambion, Austin, TX, USA) to test concentration, purity and integrity. RNA was reverse transcribed to cDNA by Reverse Transcription kit (Qiagen GmbH, Hilden, Germany). SYBR-Green Fluorescent dye (Sigma-Aldrich) was used for qPCR, performed on LightCycler (Roche Diagnostics GmbH, Mannheim, Germany) apparatus. Primers were designed according to the GenBank website, with internal reference for company, and the RNA was removed using DNase I (Ambion) Technology Co., Ltd. PCR reaction conditions were: Pre-denaturation at $94^{\circ} \mathrm{C}$ for $5 \mathrm{~min}, 94^{\circ} \mathrm{C}$ for $30 \mathrm{sec}, 63^{\circ} \mathrm{C}$ for $30 \mathrm{sec}, 72^{\circ} \mathrm{C}$ for $30 \mathrm{sec}$, a total of 35 cycles, with overall elongation for $7 \mathrm{~min}$ at $72^{\circ} \mathrm{C}$. The results were expressed by $2^{-\Delta \Delta \mathrm{Cq}}$ method.

Western blotting. Total protein was extracted by RIPA lysate (Biyuntian Biotechnology Institute, Beijing, China), and the purity was determined by BCA reagent (Wuhan Boshide
Biological Engineering Co., Ltd., Wuhan, China). Protein $(10 \mu \mathrm{g})$ was loaded, separated by $12 \%$ SDS-PAGE and transferred onto NC membranes (both from R\&D Systems, Inc., Minneapolis, MN, USA), with blocking at room temperature for $1 \mathrm{~h}$. The membranes were incubated with rabbit polyclonal PI3K antibody (dilution, 1:500; cat. no. ab182651), rabbit polyclonal Akt antibody (dilution, 1:500; cat. no. ab38449), rabbit monoclonal Bax antibody (dilution, 1:500; cat. no. ab32503), rabbit monoclonal $\mathrm{Bcl}-2$ antibody (dilution, 1:500; cat. no. ab32124), rabbit polyclonal caspase-3 antibody (dilution, 1:500; cat. no. ab13847) and rabbit polyclonal $\beta$-actin antibody (dilution, 1:500; cat. no. ab8227) (all purchased from Abcam, Cambridge, MA, USA) overnight at $4^{\circ} \mathrm{C}$. The membrane was washed three times using TBST, and secondary goat anti-rabbit (HRP) IgG antibody (dilution: 1/2000; Abcam, Cambridge, MA, USA; Catlog\#: ab6721) was added. The membrane was then incubated at room temperature for $1 \mathrm{~h}$, washed with TBST three times, and chemiluminescence (ECL; Biyuntian Biotechnology Institute) was performed. Grayscale bands were observed using an optical microscope (Olympus, Tokyo, Japan).

Statistical analysis. Using SPSS 19.0 software (SPSS, Inc., Chicago, IL, USA) for data analysis, measurement data were expressed as mean \pm standard deviation. Single factor analysis of variance (ANOVA) was used to compare indexes among all groups, and repeated measurements ANOVA was applied to compare data in each group. $\mathrm{P}<0.05$ was considered to indicate a statistically significant difference.

\section{Results}

Comparison of apoptotic rate. The apoptotic rate at each time-point in the blank control group was not significantly different. The apoptotic rate of the model and inhibitor groups increased over time, whereas that of the mimics group decreased at each time-point, and the difference in each group was statistically significant $(\mathrm{P}<0.05)$. The apoptotic rate at each time-point in the mimics group was significantly lower than that of the model and inhibitor groups, while the inhibitor group was higher than the model group, and the difference was statistically significant $(\mathrm{P}<0.05)$ (Table I).

Comparison of expression levels of mRNA in PI3K/Akt signaling pathway. The expression levels of PI3K, Akt and 
A

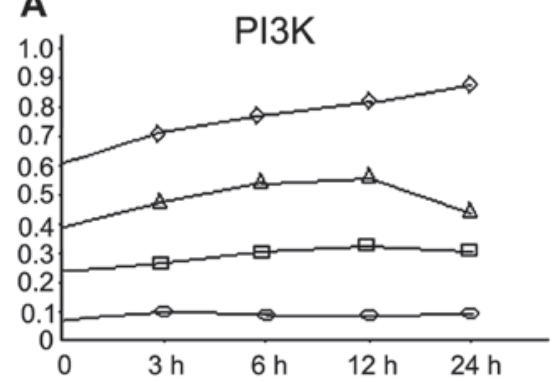

C

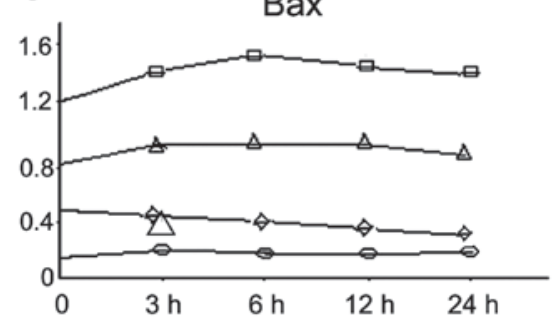

E

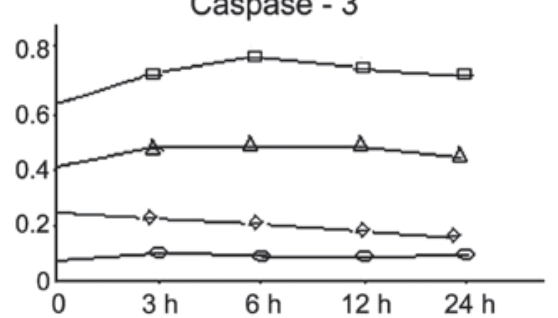

B

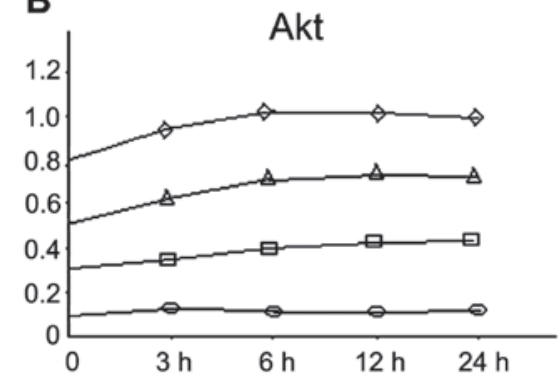

D

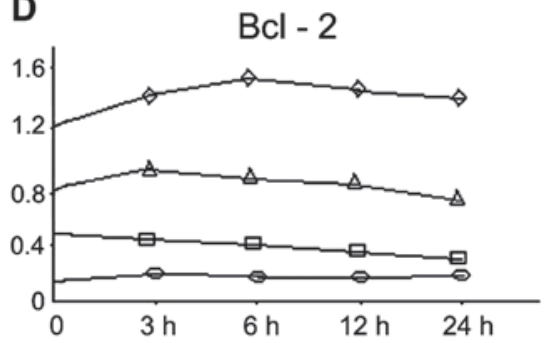

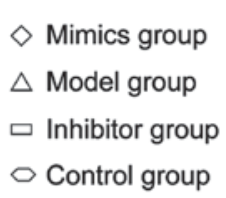

$\diamond$ Mimics group

$\triangle$ Model group

Control group

Figure 1. Comparison of mRNA expression levels using RT-PCR in PI3K/Akt signaling pathway. PI3K, phosphoinositide 3-kinase.

Bcl-2 mRNA at each time-point in the mimics group were significantly higher than those of the other groups, followed by the model group and inhibitor group, and the blank control group, which had the lowest expression levels. Differences among the groups were statistically significant $(\mathrm{P}<0.05)$. The expression levels of Bax and caspase-3 mRNA in the inhibitor group were the highest overall, followed by the model and the mimics groups, with the blank control group having the lowest expression levels. Differences among the groups were statistically significant $(\mathrm{P}<0.05)$. The changing trend at different time-points in each group is shown in Fig. 1.

Comparison of the expression level of proteins in the PI3K/Akt signaling pathway. At $12 \mathrm{~h}$, a comparison of expression level of protein in each group and among all groups coincided with the changing trend of mRNA (Fig. 2).

\section{Discussion}

The mutual intervention and regulation at different levels have led to identification of numerous transduction and regulation pathways of apoptosis. PI3K is a lipid kinase and PI3K family is related to cell proliferation, anti-apoptosis, cell migration, membrane vesicle transport, cell carcinoma transformation and other processes (8). Protein kinase B (PKB), also known as Akt, is a serine/threonine kinase. Activated Akt can regulate cell function by phosphorylating various enzymes, kinases and transcription factors. Activation of Akt phosphorylation

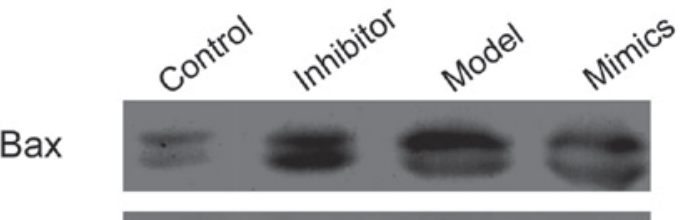

Bcl-2

Caspase-3

PI3K

Akt
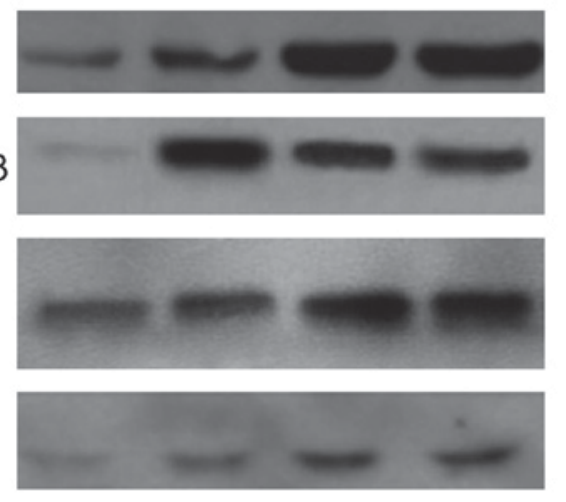

$\beta$-actin

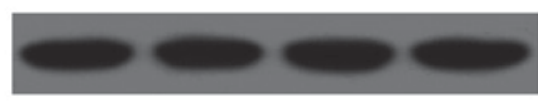

Figure 2. Comparison of protein expression levels using western blotting in PI3K/Akt signaling pathway. PI3K, phosphoinositide 3-kinase.

mainly depends on the PI3K activation, through the phosphorylation and inactivation of its downstream substrate. Activated Akt can promote cell survival via various avenues. After brain injury, multiple neurotrophic factors play a protective effect 
on the brain by activating the PI3K/Akt signaling pathway (9). Another study indicated that the Akt expression in the brain tissue of the adult rabbit was a trace, while the expression of p-Akt increased $1 \mathrm{~h}$ after traumatic brain injury, and the immune response was enhanced at the edge of the lesion (10). The difference of expression of p-Akt area in the whole brain may lead to a different extent of cell injury, suggesting that the PI3K/Akt signaling pathway participated in the regulation of the pathological process of traumatic brain injury, while the expression amount of p-Akt was related to the extent of brain injury (11). The study found that ischemic brain tissue can express erythropoietin and plays a protective role in the brain by activating ERK1/2 and Akt signaling pathways (12).

There are various expression types in the nervous system, the specific miRNAs in brain tissue contains miR-9, miR-124a, miR-124b, miR-135, miR-153, miR-183, miR-219 (13). In their study, Lagos-Quintana et al (14) found a specific expression of miR-101, miR-124, miR-127, miR-128, miR-131 and miR-132 in mouse brain tissue, with miR-124 expression being the most obvious and most highly expressed in the cerebral cortex and cerebellum (13). These miRNAs may be closely related to the differentiation of developmental neuron of brain tissue and the performance of advanced neural function (15), such as miR-124 overexpression of P19 cells of mouse which in a state of differentiation can promote axonal growth; blocking miR-124 expression could decrease the expression levels of acetylated-tubulin and affect axon growth. The expression profile of miRNAs in the tissues and plasma of patients with ischemic stroke was significant. Dharap et al (16) tested the expression of 238 miRNAs in the brain tissues of rats for $3 \mathrm{~h}$ to 3 days after the model of focal cerebral ischemia reperfusion injury was established. They found that the expression amount of 8 miRNAs increased significantly and the expression of 12 miRNAs evidently decreased. The change of miRNA expression profile after cerebral ischemia was specific, and the change of miRNAs in plasma was consistent with that in tissue (17), which indicated that miRNAs are potential markers for diagnosis of ischemic stroke.

Based on the results of the present study, we can conclude that the apoptotic rate in the model and inhibitor groups increased over time, although the mimics group decreased over time, and the difference in each group was statistically significant. The apoptotic rate at each time-point in the mimics group was significantly lower than that of the model and inhibitor groups, while the inhibitor group was higher than the model group, and the difference was statistically significant. The expression levels of PI3K, Akt and Bcl-2 mRNA and protein at each different time-point in the mimics group were significantly higher than those of other groups, followed by the model and inhibitor groups, with the blank control group was the lowest, and the difference among the groups was statistically significant. The expression levels of Bax and caspase- 3 mRNA and protein in the inhibitor group were the highest versus the model and mimics groups, while the blank control group was the lowest, and the difference among the groups was statistically significant. The results of the present study revealed that miR-124 participated in the neural protection of ischemic stroke by activating the PI3K/Akt signaling pathway, while the intervention of miR-124 or PI3K/Akt signaling pathway is a significant target for the treatment of ischemic stroke and repair of neural function.

\section{Acknowledgements}

The present study was supported by the Zunyi Science and Technology Plan, Compliance with Zunyi City Branch (2014) 78. We thank all partners and staff who helped us in the process of this study.

\section{References}

1. Taylor RC, Cullen SP and Martin SJ: Apoptosis: controlled demolition at the cellular level. Nat Rev Mol Cell Biol 9: 231-241, 2008.

2. Bergmann A: Autophagy and cell death: no longer at odds. Cell 131: 1032-1034, 2007.

3. Ebert MS and Sharp PA: Roles for microRNAs in conferring robustness to biological processes. Cell 149: 515-524, 2012.

4. Gao FB: Posttranscriptional control of neuronal development by microRNA networks. Trends Neurosci 31: 20-26, 2008.

5. Liu XS, Chopp M, Zhang RL, Tao T, Wang XL, Kassis H, Hozeska-Solgot A, Zhang L, Chen C and Zhang ZG: MicroRNA profiling in subventricular zone after stroke: miR-124a regulates proliferation of neural progenitor cells through Notch signaling pathway. PLoS One 6: e23461, 2011.

6. Zhao S, Fu J, Liu X, Wang T, Zhang J and Zhao Y: Activation of Akt/GSK-3beta/beta-catenin signaling pathway is involved in survival of neurons after traumatic brain injury in rats. Neurol Res 34: 400-407, 2012.

7. Richardson RM, Singh A, Sun D, Fillmore HL, Dietrich DW III and Bullock MR: Stem cell biology in traumatic brain injury: effects of injury and strategies for repair. J Neurosurg 112: $1125-1138,2010$

8. Gu X, Meng S, Liu S, Jia C, Fang Y, Li S, Fu C, Song Q, Lin L and Wang X: miR-124 represses ROCK1 expression to promote neurite elongation through activation of the PI3K/Akt signal pathway. J Mol Neurosci 52: 156-165, 2014.

9. Lang Q and Ling C: miR-124 suppresses cell proliferation in hepatocellular carcinoma by targeting PIK3CA. Biochem Biophys Res Commun 426: 247-252, 2012.

10. Wang ZG, Cheng Y, Yu XC, Ye LB, Xia QH, Johnson NR, Wei X, Chen DQ, Cao G, Fu XB, et al: bFGF protects against blood-brain barrier damage through junction protein regulation via PI3K-Akt-Racl pathway following traumatic brain injury. Mol Neurobiol: Dec 21, 2015 (Epub ahead of print).

11. Zhang L, Ding K, Wang H, Wu Y and Xu J: Traumatic brain injury-induced neuronal apoptosis is reduced through modulation of PI3K and autophagy pathways in mouse by FTY720. Cell Mol Neurobiol 36: 131-142, 2016.

12. Kilic E, Kilic U, Soliz J, Bassetti CL, Gassmann M and Hermann DM: Brain-derived erythropoietin protects from focal cerebral ischemia by dual activation of ERK-1/-2 and Akt pathways. FASEB J 19: 2026-2028, 2005.

13. Cheng LC, Pastrana E, Tavazoie M and Doetsch F: miR-124 regulates adult neurogenesis in the subventricular zone stem cell niche. Nat Neurosci 12: 399-408, 2009.

14. Lagos-Quintana M, Rauhut R, Yalcin A, Meyer J, Lendeckel W and Tuschl T: Identification of tissue-specific microRNAs from mouse. Curr Biol 12: 735-739, 2002.

15. Åkerblom M, Sachdeva R, Barde I, Verp S, Gentner B, Trono D and Jakobsson J: MicroRNA-124 is a subventricular zone neuronal fate determinant. J Neurosci 32: 8879-8889, 2012.

16. Dharap A, Bowen K, Place R, Li LC and Vemuganti R: Transient focal ischemia induces extensive temporal changes in rat cerebral microRNAome. J Cereb Blood Flow Metab 29: 675-687, 2009.

17. Weiss JB, Eisenhardt SU, Stark GB, Bode C, Moser M and Grundmann S: MicroRNAs in ischemia-reperfusion injury. Am J Cardiovasc Dis 2: 237-247, 2012. 